\title{
VICO: A Framework for Configuring Indoor Visible Light Communication Networks
}

\author{
Yiyang $\mathrm{Li}^{\dagger}$, Leijie Wang ${ }^{\dagger}$, Jianxia Ning ${ }^{\dagger}$, Konstantinos Pelechrinis*, Srikanth V. Krishnamurthy ${ }^{\dagger}$, Zhengyuan $\mathrm{Xu}^{\ddagger}$ \\ ${ }^{\dagger} \mathrm{UC}$ Riverside: $\{y i y l i$, lewang $\} @ e e . u c r . e d u,\{$ jning, krish $\} @ c s . u c r . e d u *{ }^{*}$ University of Pittsburgh: kpele@ $\mathrm{pitt.edu}{ }^{\ddagger}$ Tsinghua University: xuzy@tsinghua.edu.cn
}

\begin{abstract}
Visible light communications (VLC) are gaining popularity and may provide an alternative means of communications in indoor settings. However, to date, there is very little research on the deployment or higher layer protocol design for VLC. In this paper, we first perform channel measurements using a physical layer testbed in the visible light band to understand its physical layer characteristics. Our measurements suggest that in order to increase data rates with VLC (1) the beam width of a communicating link can be shrunk, and (2) the transmission beam can be tuned to point towards the target recipient. We then perform Matlab simulations to verify that the human eye is able to accommodate the changes brought by shrinking a beam or by tuning the beam direction appropriately. As our main contribution, we then design a configuration framework for a VLC indoor local area network, which we call VICO; we leverage the above features towards achieving the highest throughput while maintaining fairness. VICO first tunes the beamwidths and pointing angles of the transmitters to configurations that provide the highest throughput for each client. It then tries to schedule transmissions while accounting for conflicts and the VLC PHY characteristics. Finally, it opportunistically tunes the idle LEDs to reinforce existing transmissions to increase throughput to the extent possible. We perform extensive simulations to demonstrate the effectiveness of VICO. We find that VICO provides as much as 5-fold increase in throughput compared to a simple scheduler that does not exploit the possible variations in beamwidth or beam-angle.

Index Terms-Visible light communication, wireless indoor
\end{abstract} communication, LED.

\section{INTRODUCTION}

Recently, unlicensed optical wireless communications (OWC) have been considered as an alternative to the traditional radio frequency (RF) communications. Visible light communications (VLC) bring the promise of numerous new applications. Smart-home control messages, billboards that transmit advertisements, traffic information to users' devices, and vehicle head light based distance detection are some examples where VLC is likely to be used [3]. Going forward, one may envision that VLC can also be used to build a communication infrastructure in new generation buildings (such as in hospitals).

A VLC system consists of LED arrays (we also refer to these as simply LEDs for brevity) for transmission and photodiodes for detection. It has the following six advantages compared to RF communications: (a) VLC inherently provides increased security against eavesdroppers since it typically requires line-of-sight (LOS) transmissions and cannot penetrate walls; (b) it is harmless to humans since it operates in the visible light spectrum with much higher tolerable exposure limits; (c) VLC already enjoys ubiquitous infrastructure support. In many cases one can utilize existing lighting infrastructure to build a VLC network; (d) it is cost-efficient because its components (LEDs and photodiodes) can be massively produced and are potentially inexpensive; they can utilize the lighting infrastructure for priority "free" communications; (e) VLC is power-efficient due to the use of LEDs; (f) finally, the visible light spectrum is huge and can potentially support very high data rates. In addition, compared to traditional light sources, white LEDs have the advantages of long life expectancy, high lighting efficiency, no out-of-visible-band optical spectrum (unlike incandescent lamps which emit infrared light and fluorescent lamps which emit ultraviolet signals), easy maintenance, and environmental friendliness [4].

In this work, our focus is on an indoor VLC network. Theoretical analysis of an indoor VLC system has been presented in [5]; the paper proves the feasibility of VLC. Indoor VLC links could be classified into line-of-sight (LOS) and non-lineof-sight (NLOS) links (similar to infrared links) [6]. A directed VLC link (Fig. 1a) provides LOS communications between a transmitter $(\mathrm{Tx})$ and a receiver $(\mathrm{Rx})$. With carefully aligned transceivers LOS communications are often possible and can support high transmission speeds by focusing the transmission beam and with a narrow receiver field-of-view (FOV). With a non-directed link (Figs. 1b and 1c), the communications between a Tx with a divergent beam, and a Rx with a large FOV depend on numerous signal reflections on the surfaces in the room, instead of, or in addition to an LOS link. A link operating in the absence of LOS is defined as a diffuse link. The non-directed link is more robust to shadowing and can inherently support user mobility better. However, multipath propagation effects may limit the achievable data rate, and may require higher transmission power. In the literature ([7], [8]), it has been found that the intensity via diffuse paths is much weaker than that via LOS paths. The intensity via a LOS path is about 10 times higher than that via the first reflective path as shown in [7] in a $5 \mathrm{~m} \times 5 \mathrm{~m} \times 3 \mathrm{~m}$ room. Since LOS paths are typically feasible [7], in the rest of this paper, we only consider LOS links.

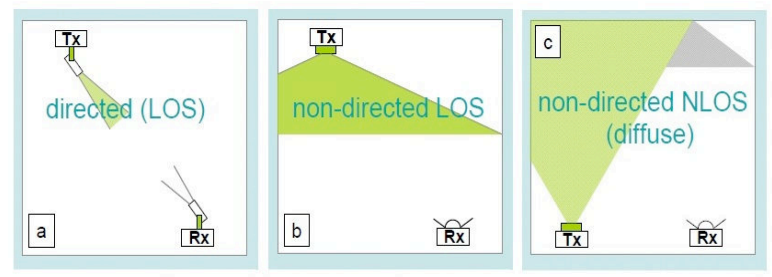

Fig. 1. Types of communication links: a) Directed line-of-sight (LOS), b) Non-directed LOS and c) non-directed NLOS (diffuse). (borrowed from [6])

Although the feasibility and advantages of indoor VLC have been previously demonstrated, higher layer protocol design for VLC is very limited. The task group under IEEE 802.15.7 [9] has been working on VLC and has drafted a medium access control (MAC) protocol which inherits most features of the one designed for wireless personal networks (WPANs); however the unique physical layer (PHY) characteristics of indoor 
VLC impact higher layer protocol design. High throughput gains are only feasible if one can account for these physical characteristics better.

Our contributions: In this paper, we first perform channel measurements and verifications in the visible light band in an indoor scenario on a real PHY layer testbed. Based on the experiments, we make the following observations: in order to increase data rate (1) the transmission beamwidth can be shrunk, and/or (2) the transmission beam can be tuned to point to target area. Based on these observations, we simulate the illumination variations using Matlab. With this we validate that the human eye is able to accommodate the changes in intensity brought about by appropriately shrinking the beam or by tuning the beam direction.

After verifying the feasibility of the two degrees of freedom (variable beamwidth and orientation of a transmitting LED), we develop a novel configuration framework (VICO for VIsible light COnfiguration framework) for an indoor VLC network. VICO schedules transmissions, and appropriately chooses the beamwidth and LED orientation, while taking into account the indoor VLC PHY characteristics. In summary, we make the following main contributions:

- Experimentation on an indoor VLC testbed: We report LOS path loss results obtained from experiments on an indoor VLC testbed. On the basis of these results, we provide insights into the PHY layer aspects that impact higher layer protocol design.

- Designing VICO: We design a novel framework VICO, for configuring an indoor VLC network. Specifically, the goal of VICO is to schedule transmissions and tune beamwidths and orientations to achieve the highest throughput under conditions of max-min fairness. We show that this problem, unfortunately is NP-hard. Thus, VICO uses a very effective heuristic to schedule transmissions. It also tunes the beamwidths and orientations to the extent possible to opportunistically increase the achieved throughput.

- Evaluating VICO: We evaluate VICO via extensive simulations using the OPNET simulator. The PHY layer characteristics are integrated to accurately characterize the VLC links. Our simulations demonstrate that VICO provides as much as a 5-fold increase in throughput compared to a scheduler that does not opportunistically exploit VLC characeristics (tuning of beamwidth and beam-angle).

Organization: The paper is structured as follows. In Section II, we discuss related work. We present our PHY layer experiments and simulations and analyze them in Section III. Our configuration framework, VICO is described in Section IV. Our extensive evaluations of VICO via simulations, are presented in Section V. We conclude our work in Section VI.

\section{RELATED WORK}

The IEEE 802.15.7 task group has drafted a MAC protocol, which inherits the properties of the one for wireless personal area networks (WPANs) [9]. In this draft, peer-to-peer, infrastructure to client and broadcast communications are all supported. The draft also considers visibility support across all topologies. Unfortunately, the draft does not account for some physical characteristics of VLC; the VLC properties (as we show) provides opportunities for increasing throughput via appropriate beam tuning. Wu et al. present a new indoor VLC system which achieves satisfactory data rates while supporting multiple access under LOS constraints [10]. The approach however is for peer-to-peer systems: It is unlikely that such communications will be commonplace in an indoor setting, more likely a local area network akin to a WLAN will be employed.

Due to similarities in propagation properties, studies on infrared (IR) are related work. In [11], the authors show that as long as the receiver FOV is larger than $25^{\circ}$ there is very little chance for a communication link to be blocked in an indoor setting. Based on a shadowing analysis versus ceiling height (where lights are mounted), the authors conclude that the probability of shadowing is low; in particular they show that the likelihood of link shadowing is lower in rooms with larger ceiling heights. Given these results, we assume that shadowing problems can be ignored in the setting considered.

Charoen Tangtrongbenchasil designs an indoor ubiquitous optical free space communication system in [12]. This system is composed of a Tx array board on which each LED emits light through a lens to cover an area (called a cell) on the ground; overlaps across cells can be controlled by an electronic switching matrix hardware. The hardware also contains a collocated Rx array which can appropriately collect received light. Therefore, highly accurate and well controlled directionality can be achieved. This system is able to obtain knowledge of user devices' locations so that a coordinator can stimulate LEDs to transmit signals to desired receiver devices. In this paper, we adopt a similar configuration for our system; we depict the same in Fig. 2. As seen in Fig. 2, in order to cover the entire room, we enforce overlaps between each pair of adjacent cells in the default setting. Intuitively, the users located in such overlapped areas have a high possibility of being interfered with. Therefore, when we assigning resources, the interference effect should be carefully taken into account.

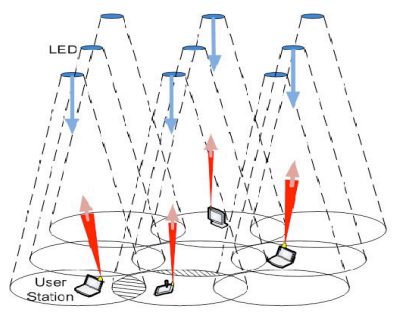

Fig. 2. An indoor VLC Network: LED arrays are mounted on the ceiling. There are 9 cells in the realization. The clients are all at desk-top height.

The conflict graph approach has been used in the RF context to schedule interference-free transmissions (e.g. [13], [14]) The vertices in the conflict graph are potential communication links in the original graph (with the real nodes and edges); an edge connects two vertices if the corresponding two links in the original graph, cannot be active simultaneously. While the basic approach that we use for the scheduling component within VICO is similar, we account for the PHY layer characteristics of VLC when constructing the conflict graph. More importantly, VICO facilitates beam tuning unlike in the prior efforts.

\section{Physical Layer Characteristics of VLC}

We perform channel measurements to obtain a coarse understanding of the VLC PHY layer, using a simple testbed in an indoor scenario. As alluded to earlier, our main findings are: (1) the beamwidth can be adjusted and it is inversely 




Fig. 3. Visible light experimental testbed.

proportional to achievable data rate; (2) the transmission beam can be tuned to directionally (not vertically) illuminate an adjacent area; the path loss is proportional to the beam-angle ${ }^{1}$. Following these findings, we perform simulations to examine the illumination variations when we change beamwidth or beam-angle. We find that these variations do not hamper visibility to the human eye.

Experimental setup: In our experiments, we employ a single LED as the transmitter, and a photodiode as the receiver as shown in Fig. 3. At the transmitter, we use a 1 Watt light spot on "OptoTherm Linear Heat Spreader with Lens" white LED (OPA742W23). The illumination output of the LED is $41 \mathrm{~lm}$ with a driving current of $350 \mathrm{~mA}$ and a full beam angle of $23^{\circ}$. The relative radiant power spectral density and the spatial intensity distribution curves of each LED are available in the data-sheets [15]. On the receiver side, a commercial photodiode (PDA10A) with an internal trans-impedance preamplifier is used. It has an active area of $0.8 \mathrm{~mm}^{2}$ and an electrical signal bandwidth of $150 \mathrm{MHz}$ [16]. An optical bandpass filter (Thorlabs FB450-40) is added in front of the photodiode. The filter has a center wavelength of $450 \mathrm{~nm}$, a full width at half maximum (FWHM) of 40nm, and transmittance of $70 \%$ [17]. These components are jointly used to expand the active receiving area and reduce the ambient light noise. The receiver acts as an energy detector and can detect signals using on-off keying (OOK) or pulse position modulation (PPM).

\section{A. Beamwidth adjustment}

We conduct experiments to understand the relation between the transmission beamwidth and the area of coverage with LOS communications. The area of coverage will decrease if we reduce the beamwidth and the intensity of the shrunk-down area will increase for a fixed transmission power. There are two ways to alter the energy intensity: changing beamwidth or varying the distance between the Tx-Rx pair. Since we do not have access to LEDs with adjustable beamwidths, we instead vary (reduce or increase) the Tx-Rx distance to mimic varying the beamwidth (shrink or expand the illumination area). Consider as in Fig. 4, a LED mounted on the ceiling at point $T$. If the beamwidth is $\theta_{1}\left(\theta_{i}\right.$ reflects half the beamwidth), the coverage area at $h_{1}$ (the distance from the LED) is $C_{1}$. If we shrink the beamwidth to $\theta_{2}$, the coverage area is $C_{2}$. The energy is uniformly distributed in the plane when the plane is orthogonal to the transmission beam. The energy intensity is the same on different intersection circles as long as the size of the circle is equivalent. When the area of $C_{2}$ and $C_{3}$ are

\footnotetext{
${ }^{1}$ We define beam-angle as the angle between the axis of transmission beam and the vertical axis.
}

the same $\left(C_{2}\right.$ corresponds to beamwidth $\theta_{2}$ at distance $h_{1} ; C_{3}$ corresponds to beamwidth $\theta_{1}$ at distance $h_{2}$; the area of $C_{2}$ is identical to the size of that of $C_{3}$ ), the energy intensity in $C_{2}$ is equivalent to that in $C_{3}$; this is true as proved in [18], based on the scaling property of illumination rendering. It was found that the basic illumination pattern at $k \times h$ ( $\mathrm{k}$ is a constant) is identical to that at $h$ with its beamwidth scaled up by $k$. This is because with a shrinking beamwidth there is a decrease in path loss; this decrease counterbalances the increase in path loss brought about by the increased distance. The relationships between the variables in Fig. 4 are shown as follows:

$$
\begin{aligned}
& x_{1}=h_{1} * \tan \left(\theta_{1}\right) \\
& x_{2}=h_{1} * \tan \left(\theta_{2}\right)=h_{2} * \tan \left(\theta_{1}\right)
\end{aligned}
$$

From the above, we have

$$
x_{1} / x_{2}=\tan \left(\theta_{1}\right) / \tan \left(\theta_{2}\right)
$$

We consider an LED transmission beamwidth specified by $\theta_{1}=18^{\circ}$ in the measurement settings ${ }^{2}$. Without loss of generality, the term "beamwidth" in the rest of the paper refers to this half beamwidth angle $\theta_{1}$. We test three scenarios at distances of $2 \mathrm{~m}, 2.5 \mathrm{~m}$ and $3 \mathrm{~m}$, respectively. These distances correspond to the $h \mathrm{~s}$ in Fig. 4. From (3), we have $x_{1}^{2} / \tan ^{2}\left(\theta_{1}\right)=x_{2}^{2} / \tan ^{2}\left(\theta_{2}\right)=x_{3}^{2} / \tan ^{2}\left(\theta_{3}\right)=A$. Having the values of $x_{\{1,2,3\}}$ and $\theta_{1}$, one can derive that $\theta_{2}=15.18^{\circ}, \theta_{3}=12.28^{\circ}$, and $A=8.91$. In these three scenarios, the energy intensity is measured by a power meter as: $1.89 \mu \mathrm{W}, 1.13 \mu \mathrm{W}$, and $0.85 \mu \mathrm{W}$ correspondingly. This shows that for a fixed beamwidth, the farther from the LED, the lower the energy intensity that is achieved. The measurements thus show that the energy intensity is inversely proportional to the area covered [18].

The energy intensity directly affects the perceived signalto-noise ratio (SNR). Assuming that the background noise is white Gaussian with a constant mean, the perceived SNR is proportional to the energy intensity [4]. As the intensity can be considered as power, the intensity (SNR) and data rate are related [1]. As the SNR increases, the data rate will increase. As shown in [2], the achieved increase in data rate is dependent both on the delay spread of the environment and the target BER of the application of operation. In high SNR regimes, needed for achieving extremely low BERs, it is shown that small increases in SNR can lead to a two-fold reduction in the supportable bit duration with OOK. Higher SNR increases may be needed in low SNR regimes to achieve the same reduction. Since we do not have exact measurements of the delay spread of the setting and the corresponding SNR increases needed to increase the rate (decrease the bit duration), we make a very conservative assumption that doubling the data rate requires a two-fold increase in the SNR in our simulation experiments reported in Section V. Note however, that our proposed framework is not dependent on this assumption. In fact, if the SNR increases needed to double the rate are much lower (as reported in [2]), our approach can yield even higher throughput benefits.

With the assumption that the data rate is proportional to the $\mathrm{SNR}$, it is proportional to the received energy intensity and inversely proportional to the coverage area size. Assuming that

${ }^{2}$ The maximum beamwidth of the LED in use as shown in its data-sheets [15] is $23^{\circ}$. This means that the energy is uniformly distributed within the area covered by this full beamwidth of $23^{\circ}$. However, our measurements suggest that the total energy loss occurs (i.e., it is totally dark) beyond the edge of the area covered by a beamwidth specified by $\theta_{1}=18^{\circ}$. 


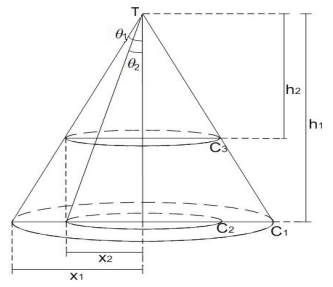

Fig. 4. The relationship between beamwidth and distance from the LED source.

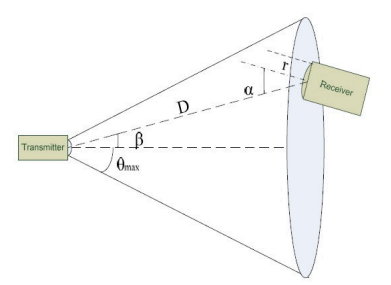

Fig. 5. LOS channel model (from [4])

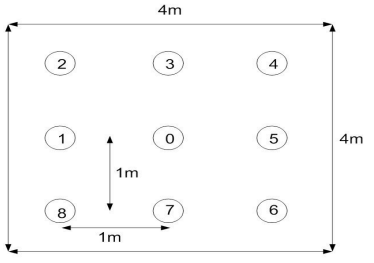

Fig. 6. LED distributions in a $4 \mathrm{~m} \times 4 \mathrm{~m}$ room. Each LED has been labeled; LED 0 is the coordinator.

TABLE I

ILLUMINANCE COMPARISON IN A ROOM WITH BEAMWIDTH AND BEAM-ANGLE ADJUSTMENTS

\begin{tabular}{|l|l|l|}
\hline Scenarios & Average SNR (dB) & the minimum SNR (dB) all-over the room \\
\hline \hline No adjustments (See left plot in Fig. 7) & 61.556 & 51.3923 \\
\hline Beamwidth adjustment (See right plot in Fig. 7) & 61.9226 & 51.3923 \\
\hline Beam-angle tuning 1 (See left plot in Fig. 9) & 61.548 & 51.3923 \\
\hline Beam-angle tuning 2 (See right plot in Fig. 9) & 61.4019 & 51.3923 \\
\hline
\end{tabular}

an on-off-keying (OOK) modulation scheme is adopted, we have $R_{1} / R_{2}=E_{1} / E_{2}=x_{2}^{2} / x_{1}^{2}=\tan ^{2}\left(\theta_{2}\right) / \tan ^{2}\left(\theta_{1}\right)=K$, where $R_{i}$ is the data rate achieved with a coverage area $C_{i}, E_{i}$ is the energy or intensity with the same coverage area $(i \in\{1,2\})$, and $K$ is a constant [18]. If we want to double the data rate, we have to shrink the beamwidth to $12.9^{\circ}$ (from $18^{\circ}$ ). At desk-top height $(0.5 \mathrm{~m})^{3}$, the radius of the coverage area is $0.8123 \mathrm{~m}$ when the beamwidth is specified by $\theta_{1}$ equal to $18^{\circ}$. Therefore, in order to cover a room with $4 \mathrm{~m} \times 4 \mathrm{~m}$, we need at least 9 LEDs with minimal overlaps between each service cell. Adjacent LEDs are separated by a space about $1 \mathrm{~m}$. Please note that the specific settings are for OOK modulation and obtained from our experimental testbed in Fig. 3.

Matlab simulations: Based on the LOS path loss model in (4) of [4], the illuminance distribution in a $4 \mathrm{~m} \times 4 \mathrm{~m}$ room has been simulated using Matlab. In (4), $m$ is the order of Lambertian emission ${ }^{4}$ and is related to the semi-angle at the half illuminance of an LED $\Phi_{1 / 2}$; specifically, $m=$ $-\ln 2 / \ln \left(\cos \left(\Phi_{1 / 2}\right) . P_{r}\right.$ and $P_{t}$ denote the received power and the transmitted power, respectively. The angle between the sourcereceiver line and the receiver orientation (see Fig. 5) is $\theta$; the angle between this line and the source beam axis is $\beta$ (viewing angle). As shown in Fig. 5, if $\theta$ is larger than the receiver's FOV, the LOS link does not exist and therefore cannot receive from the target LED. $\theta_{\max }$ is the maximum beamwidth. $\Omega_{r}$ is the Rx solid angle ${ }^{5}$ seen by the Tx and $A_{r}$ is the Rx area. They satisfy the relationship $A_{r} \cos (\theta)=D^{2} \Omega_{r}$. The Rx distance to the source is $D$. Some of these relationships are illustrated in Fig. 5. For more details the reader is referred to [4].

$$
\text { Pathloss }=\frac{P_{r}}{P_{t}}=\frac{(m+1) A_{r}}{2 \pi D^{2}} \cos (\alpha) \cos ^{2}(\beta) .
$$

The simulation settings are as follows: there are $9(3 \times 3)$ LED arrays uniformly mounted on the ceiling. The specific deployments are shown in Fig. 6. Each array consists of $100 \times 100$ individual LEDs. In the simulation, we set $\theta_{\max }=18^{\circ}$, which is consistent with what was used in our experiments. The receiver's FOV is $60^{\circ}$. The photodiode's receiving area $A_{r}$ is $1.0 \mathrm{~mm}^{2}$. Other parameters are set identical to those in

\footnotetext{
${ }^{3}$ Normally, we assume the room height is $3 \mathrm{~m}$; thus the distance from the LED to a node at desk-top height is $2.5 \mathrm{~m}$.

${ }^{4}$ Lambertian emitter is a light source whose radiance varies according to Lambert's cosine law.

${ }^{5}$ The solid angle, $\Omega_{r}$, is the two-dimensional angle in three-dimensional space that an object subtends at a point.
}
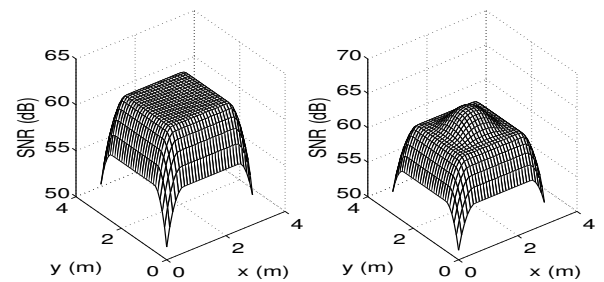

Fig. 7. Illuminance distribution in a $4 \mathrm{~m} \times 4 \mathrm{~m}$ room. Left: all LEDs are facing vertically to the ground with identical beamwidths of $18^{\circ}$ (default deployments in which all the LEDs are at default positions); Right: the middle LED array's beamwidth is shrunk to $12.9^{\circ}$.

[5]. We compute the illumination at desktop height using (4), assuming that all the LEDs are facing vertically downwards (towards the ground) and have the same beamwidth. The illuminance distribution is plotted on the left side in Fig. 7. If we shrink the middle LED array's beamwidth to $12.9^{\circ}$, the illuminance distribution is shown in the right plot. From the plots, we can see the SNR increase in the middle of the room and the corresponding decrease at the edges. The maximum SNR for left scenario is $63.0062 \mathrm{~dB}$ and $66.0712 \mathrm{~dB}$ for right one.

\section{B. Beam-angle tuning}

Next, we examine if we can tune the pointing direction (beam-angle) of a transmission beam to achieve better performance. For example, in Fig. 8, the shaded area to the bottom left is covered by two LED arrays. If the two LED arrays transmit the same information to the node in this area simultaneously (assuming OOK), the SNR will increase and so does the achievable data rate. However, if these two LED arrays send different signals at the same time, it would cause severe interference. We have to take these into account in higher layer protocol design.

Consider the case where adjacent LED arrays send the same information. At the target Rx, the time delay between the two paths from two LED arrays depends on the difference in the distances on the two paths. In an indoor VLC system, the distance between two LED arrays is of the order of a few meters; the distance from ceiling to the desk-top height or ground is again of the order of a few meters. Typically the maximum distance difference on the two paths from the pair of LEDs to the user is typically no more than 5 meters and the corresponding time delay is about $1.6 \times 10^{-8} \mathrm{~s}$. Compared 


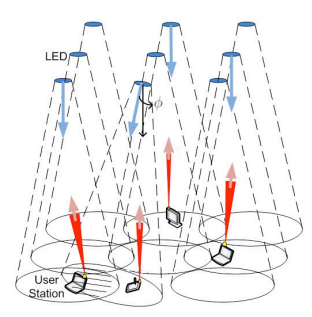

Fig. 8. An indoor VLC network: One of the 9 LED arrays has been tuned to illuminate the adiacent cell.

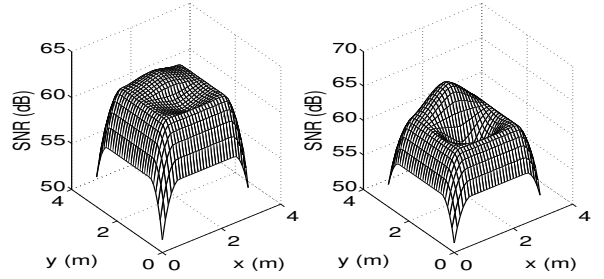

Fig. 9. Illuminance distribution in a $4 \mathrm{~m} \times 4 \mathrm{~m}$ room. The middle LED array is tuned to directionally shift towards the top-middle cell. Left: the center of the coverage of the tuned beam-angle is $0.1 \mathrm{~m}$ away from default position; Right: $0.5 \mathrm{~m}$ away from default position.

to the LED's modulation bandwidth which is $2 \mathrm{MHz}$, the time delay is negligible. Thus, we can ignore the impact of this delay spread with minimum loss in accuracy.

Matlab simulations: We follow similar simulation procedures and use settings as in subsection III-A. Our goal is to observe the illuminance changes due to the tuning of the beamangle. In Fig. 9, we see that the maximum SNR is increased in the cell towards which the middle LED array is redirected. The SNR variation depends on the extent to which the beam-angle of the LED array is tuned. For example, when the middle LED array's beam-angle is tuned such that the coverage area shifts upwards by $0.1 \mathrm{~m}$ from the default position (left plot in Fig. 9) the maximum SNR increase is $1 \mathrm{~dB}$ in the middle cell of the top row. Correspondingly, the SNR in the bottom middle cell decreases by about $2 \mathrm{~dB}$. When the middle LED array beam shifts by $0.5 \mathrm{~m}$ from the original position (right plot in Fig. 9), the maximum SNR increase in the top middle cell is $3 \mathrm{~dB}$ and the decrease in SNR in the bottom middle cell is about $8 \mathrm{~dB}$.

\section{Eye sensitivity to the beam adjustments}

The illumination difference brought by beamwidth adjustments and beam-angle tuning directly impacts a human's feeling on the illumination. Since the international LED lighting standards is still under development [19], the sensitivity of human eyes to the illuminance change brought by tuning beamwidth and beam-angle has not been studied extensively. However, it is generally believed that a change of about 1 to $3 \mathrm{~dB}$ in the average and minimum SNRs (illumination) is unlikely to significantly affect the perception of a human. We assume that the left plot in Fig. 7 which represents the default case (all the lighting sources are distributed uniformly and point vertically to the ground), corresponds to a scenario of acceptable illuminance. Note that in our simulations, the SNR directly corresponds to the illuminance. The maximum SNR in the default case is $63.0062 \mathrm{~dB}$, the average SNR $61.556 \mathrm{~dB}$ and the minimum SNR $51.3923 \mathrm{~dB}$. The minimum SNR is observed at the four corners of the room. In Table I we present the average and the minimum SNR values with the different approaches considered (default, with beamwidth adjustment, and with beam-angle adjustments). We see that in all cases, the change in the average is very small $(<1 \mathrm{~dB})$; the minimum SNR does not change at all. This suggests that tuning the beam-width and the beam-angle as considered, does not impact the illumination in the room by much.

Discussion: Our current experimental set up is rudimentary since it is one of the very first VLC experimental set ups that we know. As the technology evolves, and we progressively improve our experimentation capabilities, we expect that many of the functions that we consider (e.g. varying the beam-angle) will be possible in real deployments.

\section{DESIGN OF VICO: OUR CONFIGURATION FRAMEWORK}

In this section, we present the design of our configuration framework VICO. In a nutshell, the goal of VICO is to schedule transmissions in the VLC network so as to achieve the highest throughput under conditions of max-min fairness. In achieving this goal, VICO attempts to exploit both beamwidth adjustments and beam-angle tuning to opportunistically increase throughput.

In a nutshell, VICO first allows clients to associate with their closest LED array (which are similar to base stations); this allows the LED array to determine the location of the client. The LED array then finds the best beamwidth and beam-angle for each client. The information is then fed to a central controller that then makes the scheduling decisions; it determines which LED arrays can be active simultaneously in a time slot and which clients they serve. Finally, the controller also triggers unused LED arrays to enhance the signal quality (and hence the rate) at clients that are already being served (send the same information as the default LED array that is already serving the client), if possible. The centralized controller makes it easy for the LED arrays to be synchronized and we assume as such in this work. A block diagram depicting the functionalities of VICO is in Fig. 10.

Each LED array communicates with a single user (or client) at any given time; note however that clients can receive simultaneously from two arrays if they transmit the same information. If there are multiple users associated with a LED, the transmissions to the users are separated in time (each user is served in a different time-slot). Our goal is to then determine the set of clients, that can be simultaneously served in a single time slot.

We use a conflict graph to model the effects of interference. The conflict graph determines the groups of links that mutually interfere and hence, cannot be active simultaneously. Two links are said to interfere with each other if (a) they are within the coverage of two or more LEDs and (b) the LEDs are transmitting different data (to different clients). Based on the conflict graph, VICO attempts to do the following: (1) create groups of links that are conflict free (these are links that can be simultaneously activated); since groups of links are activated one by one, fairness is ensured (2) maximize the throughput by minimizing the number of groups; (3) opportunistically increase throughput by utilizing inactive LEDs to increase SNR at clients already being served (on a "water-filling" basis).

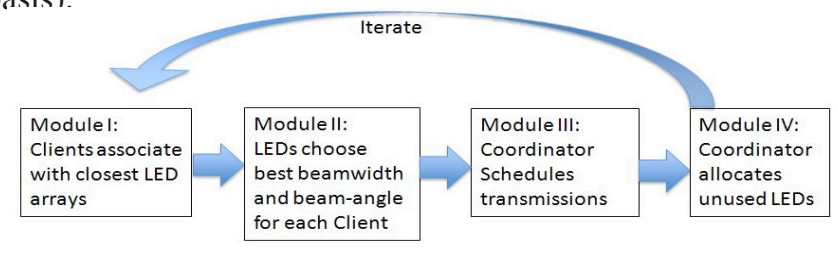

Fig. 10. A high level overview of VICO 


\section{A. Scheduling problem formulation}

In order to avoid conflict, we seek to partition the network into different sub-groups. The links in the same group share the medium without interfering with each other. Let us assume that $M$ LED arrays and $N$ clients are in the room. For ease of discussion, we assume that $N>M$ (as typical in a conference room or a hospital lounge); VICO however, is applicable when $N \leq M$. Let $x_{i j}=\left(l_{i}, c_{j}\right)$ be the link between LED $i$ and client $j$. Two links, $x_{i j}$ and $x_{p q}$ that interfere with each other cannot be scheduled in the same time slot. Our goal is to create the minimum number of groups, each of which contains a set of non-interfering links. In each time slot, a specific group is activated. If we are able to minimize the number of groups, we achieve maximum throughput under max-min fairness conditions. We first show that our problem is NPhard. Subsequently, we design an efficient heuristic to create the groups.

Theorem 1: The scheduling problem for our VLC system which has two degrees of freedom (beamwidth and beam-angle of each LED) is NP-hard.

Proof: We first show that the problem with fixed beamwidths and angles (let us call it Problem 1) is NP-hard. We define the conflict (interference) graph $G=(V, E)$, where the set of vertices $V$ is the set of possible links $x_{i j}$ in the original network graph. An edge $e \in\{E\}$, between two vertices in the interference graph exists if the two links (vertices) interfere with each other. Since the two vertices (links) that are connected with an edge in the interference graph conflict with each other, they need to be scheduled in different time slots and thus be colored differently. In other words, we want to color the vertices of graph $G$ in such a way that no two adjacent nodes share the same color. This is the classic vertex coloring problem which is known to be NP-hard [20].

In our general problem, we have two additional degrees of freedom (beamwidth and beam-angle of each LED). This means that we no longer have only one interference graph. Consequently, we need to find a coloring with the minimum chromatic number across all possible interference graphs. Since Problem 1 which is a special case of our problem is NP-hard, we conclude that our problem is also NP-hard. In other words, even if we had a way to find the beamwidths and the orientations of every LED that leads to the "best" throughput, we would not be able to determine the schedule since problem 1 is NP-hard.

Given the result in Theorem 1, we next propose a heuristic to group non-conflicting links as efficiently as possible.

\section{B. LED array regulations}

In order to simplify the problem and consider feasibility, we assume that each LED can only have two different beamwidth configurations (denoted as $\theta_{0}$ and $\theta_{1}$ ) and three options for beam orientation (with corresponding beam-angles $\phi_{0}, \phi_{1}, \phi_{2}$ ). The beamwidths are $18^{\circ}$ and $12.9^{\circ}$ respectively, to be consistent with our PHY layer studies. The three different beam orientations can be achieved in four directions (to reach out to possible adjacent neighbor cells). $\phi_{0}$ represents the angle where the LED is facing the ground vertically i.e., $\phi_{0}=0^{\circ}$. We refer to this pointing position as the original (default) position. The beam-angle $\phi_{2}$ is the offset angle from original position when the coverage area completely overlaps with an adjacent neighbor cell when the latter's beam is set in the original position. The beam-angle $\phi_{1}$ creates a half-way overlap i.e., $\phi_{1}=\frac{1}{2}\left(\phi_{2}\right)$.

Scope: The fixed number of beamwidths and beam-angles are chosen to simplify the description of our solution and our evaluations. Moreover, they also tie in with our PHY layer studies described in Section III. One would only expect a finite set of beamwidth and beam-angle combinations used since the number of data-rates possible belong to a finite discrete set (based on the modulation and FEC combinations used). VICO is applicable in such more general cases with no modifications. If electronic steering is possible and beamwidths and beamangles can be tuned to arbitrary values, VICO will still apply.

\section{Details of VICO}

The following modules make up our framework VICO. Again, these modules and the sequence of functions in VICO are depicted in Figure 10. Given that the scheduling problem with the optimal beamwidth and beam-angle is NP-hard, we use a heuristic to group the non-conflicting links within the same time slot in the third module.

Module I (Client Association): Each client chooses the nearest LED array to associate with. A LED array may have multiple associated clients. We assume that clients can determine the closest LED based on beacons that are transmitted during a calibration phase. Since we only care about scheduling downlink transmissions in this paper, we assume that an LED array can determine the location of a client with the received signal or from an image sensor [4]. Alternatively, clients may use localization methods (e.g. [22]) to determine their coordinates and this is later communicated to the LED array.

Module II (LED Tuning): Each LED array chooses the best combination of beamwidth and beam-angle from sets $\left\{\theta_{0}, \theta_{1}\right\}$ and $\left\{\phi_{0}, \phi_{1}, \phi_{2}\right\}$ for each associated client. The "best" combination is the one that minimizes path loss. Once an LED array obtains the coordinates of an associated node, it calculates the path loss to the node with different combinations of beamwidth and beam-angle. It then chooses the combination that yields the lowest attenuation and hence the highest SNR; this in turn allows the LED array to transmit with the highest viable rate to the client. In the case of ties, the default position $\left(\phi_{0}\right)$ and the smaller beamwidth $\left(\theta_{0}\right)$ are used. This is because communications with this combination has the low associated possibility of interfering with neighbor cells.

Module III (Scheduling Transmissions): Next, the information with regards to each client is fed back to a central controller. The controller generates a basic conflict graph with the information provided. This conflict graph $G=(V, E)$ contains vertices that correspond to the links determined in previous module (with the corresponding beamwidth and beam-angle). There is an edge between the vertices $x_{i j}$ and $x_{p q}$ in $G$ if the links $x_{i j}$ and $x_{p q}$ cannot be active simultaneously; $i, p$ denote the LEDs' labels and $j, q$ represent the nodes' labels. To begin with the controller tries to find the group of links $S_{0}$, which are to be scheduled in the first slot. Note here, that since each LED can at most serve one client at a time, the maximum size of $S_{0}$ is the number of LED arrays in the network. It picks a link from $G=G^{(0)}$ at random (say the link $x_{i j}$ ) to be scheduled in this slot i.e., the link is included in $S_{0}$. It then picks a second link (not picked before) at random; if this link interferes with the links that are already included in $S_{0}$, it is eliminated; else, it is included in $S_{0}$. The process is repeated 
for each link until 1) all links are either included in $S_{0}$ or eliminated or 2) the number of links in $S_{0}$ has reached the maximum value (whichever condition is met first). The group of links in $S_{0}$, are scheduled in the first time slot.

Next, all the links that were included in $S_{0}$ are removed from consideration (nodes are removed from the conflict graph) to form $G^{(1)}$. The process in the above paragraph is repeated for $G^{(1)}$ to form the next group $S_{1}$. The grouping procedure itself is repeated (for each $G^{(k)}$ ) by eliminating links that were included in prior groups until no links are left.

One can envision opportunistically scheduling links that were scheduled in previous groups, in later groups if possible. For example, one can envision a link scheduled in $S_{0}$ being again scheduled in $S_{1}$, after all the previously unscheduled links were considered for $S_{1}$. Instead of this, here, we seek to reinforce transmissions at the clients that receive information in $S_{1}$, using the LEDs that are idle. We find that this allows the clients to receive data at higher SNRs and therefore at higher bit rates.

The steps of the process are formalized with the pseudocode below:

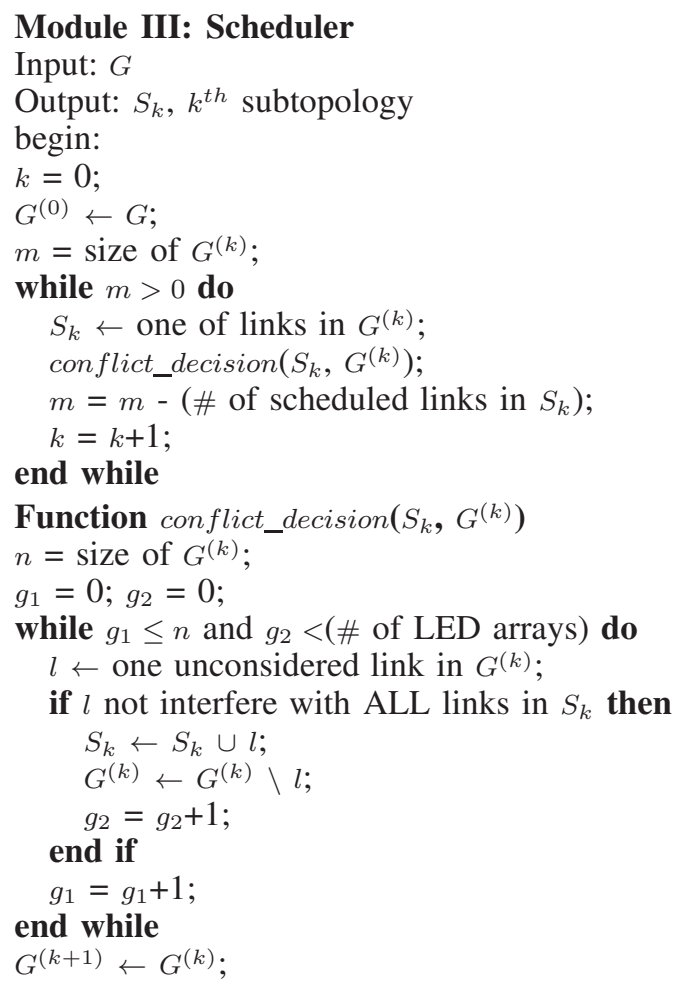

Module IV (Reinforcement via Idle LEDs): In each time slot, due to conflict, many LEDs may only illuminate without sending any meaningful information. These silent LEDs can be used to reinforce (provide additional power) active links. An idle LED array would change its beam-angle and choose an appropriate beamwidth to send information to a client that is currently being served by a different LED. The approach is based on the "water-filling" principle, which works if one were to use an energy detector using OOK modulation scheme. Since the data rate is related to the achievable SNR the approach is used to increase SNR. We first choose the client with the lowest data rate to receive additional power from the nearest silent LED array (if possible). This procedure is repeated until all the silent LED arrays have been assigned to serve one of the active clients (or are categorized as "cannot be used for doing so").

\section{Evaluations of VICO}

We evaluate the performance of VICO via extensive simulations. Specifically, we compare the performance of scheduling transmissions with and without VICO. In the cases without VICO, we set the LED beamwidths to fixed default settings; further, the beam-angle is not tunable. For simplicity, we assume that by using prior indoor localization methods each client is aware of its location and coordinates within the network [21], [22]. This information is then sent via explicit control messages to the central coordinator with VICO. In all our simulations, we use the pathloss models in Section III. In addition, we input the LEDs' configurations in the specific scenarios considered into a Matlab program in order to check the illuminance distributions as in Section III.

\section{A. Simulation setup}

We implement VICO using OPNET 16.0.A [23]; we provide the details below.

Phases of operations: We implement all the functional modules within VICO. Specifically, we divide the operations into four phases. Each of the phases roughly corresponds to a specific module within VICO.

- Phase 1: Each client identifies the LEDs that it can communicate with. It then sends an association message indicating its location, to the closest LED.

- Phase 2: Each LED array sends the information with regards to its associated clients and their locations to the coordinator. The coordinator determines the optimal transmission beamwidth and beam-angle for each client.

- Phase 3: The coordinator assigns links to time slots as per the heuristic-based approach of VICO discussed in Section IV. It also identifies idle LEDs and determines if they can reinforce transmissions to clients that are already being served.

- Phase 4: The coordinator notifies each LED about the transmission schedule. Then, each LED sends information to each client as per the schedule; the beamwidth and beam-angle are appropriately tuned specific to the client to which the transmission is performed.

Parameter settings: The experimental parameters in Section III are also used in the simulations. As mentioned, we adopt the channel model in (4). The specific settings are provided in Table II. The simulation setting is a room which is 4 meters long, 4 meters wide and 3 meters high. All the users are located at a height of 0.5 meters. Each adjustable LED can use two beamwidths, viz., $12.9^{\circ}$ and $18^{\circ}$; it can also use four pointing directions and two steps in each direction (to define the beam-angle as discussed in Section IV). We use a basic data rate of $1 \mathrm{Mbps}$. The data rate increases in proportion to the SNR (if the SNR is doubled, the data rate increases to $2 \mathrm{Mbps}$ and so on). The default SNR for supporting the basic rate is assumed to be $61 \mathrm{~dB}$. In order to double the data rate, this SNR has to increase by $3 \mathrm{~dB}$. This again, is a conservative scaling of the data rate relative to SNR changes as discussed in Section III. Our framework can apply regardless of how the data rates change with SNR and if the operations are in very high SNR regimes (as in [2]), the improvements will be even higher than that reported here. Scenarios: We primarily compare the case with VICO with the cases where (a) one degree of freedom 


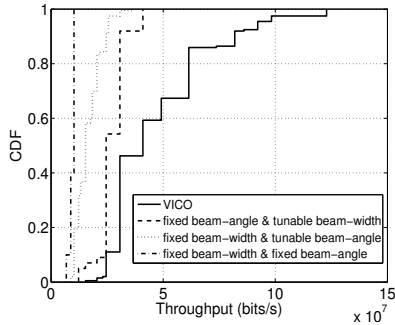

Fig. 11. CDFs of the throughputs with the four approaches: VICO provides significant gains over other approaches.

TABLE II

SIMULATION SETTINGS

\begin{tabular}{|l|l|}
\hline \# of directions & 4 \\
\# of beamwidth & 2 \\
Basic data rate & $1 \mathrm{Mbps}$ \\
Traffic pattern & $10000 \mathrm{pkt} / \mathrm{sec}$ \\
Packet size (association) & $48 \mathrm{bits} / \mathrm{pkt}$ \\
Packet size (data and control) & $32 \mathrm{bits} / \mathrm{pkt}$ \\
Network size & $4 \mathrm{~m} \times 4 \mathrm{~m} \times 3 \mathrm{~m}$ \\
\hline
\end{tabular}

(only beamwidth or beam-angle) can be adjusted and (b) where no degree of freedom can be adjusted. We vary the client density in the network to examine the performance of VICO. In a small scenario, we perform an exhaustive search with all possible beamwidths and beam-angles and find the maximum throughput achieved (by consuming the minimum number of time slots). We compare that with the performance with VICO to examine how close the configuration of VICO is likely to be with respect to the optimal. Finally, we also examine the impact of reinforcement with idle LEDs.

Metrics: We evaluate VICO in terms of network throughput, number of time slots that are consumed to accommodate all links and the illuminance distributions. Each scenario is simulated at least 20 times and a statistical average is computed. An exception is the case where we examine the illuminance; here, we select a single scenario randomly.

\section{B. Results and Discussion}

VICO is efficient in terms of network throughput and the number of time slots consumed for scheduling links: We compare the total network throughput with four different scenarios; (1) With VICO where we exploit both degrees of freedom (tunable beamwidth and beam-angle); (2) With one degree of freedom (tunable beamwidth but fixed beam-angle); (3) With one degree of freedom (tunable beam-angle but fixed beamwidth); (4) With fixed beamwidth and beam-angle. The total number of clients within the network is 20 .

In Fig. 11, we plot the CDFs of the throughputs with the four scenarios. The average throughputs are shown in Fig. 12. It is easy to see that VICO almost doubles the median throughput compared to a case where only the beamwidth is adjustable. There is about a three-fold increase in the average throughput compared to the case where we only tune the beamangle and the beamwidth is fixed at $18^{\circ}$. The improvement is about 5 fold if the LED has no freedom in terms of adjustment i.e., compared to a vanilla scheduler. These results demonstrate the efficacy of VICO in exploiting the degrees of freedom to provide a drastically increased throughput.

Next, we examine the number of time slots consumed in order to schedule all the links in the network with the above four approaches. We again perform 20 simulation runs for each

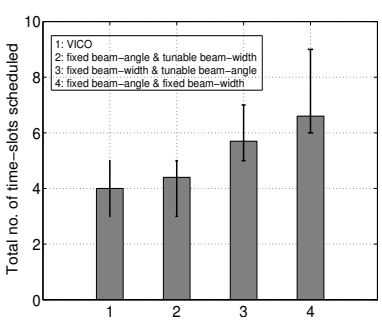

Fig. 13. Total number of time slots required for scheduling all links with the four scenarios. case and compute the average number of time slots used. For the four cases, the average number of consumed time slots are 4, 4.4, 5.7, and 6.6, respectively, as shown in Fig. 13. Intuitively, the fewer the time slots the more the clients that are included in each possible group. This implies that a higher number of simultaneous active transmissions are possible and this again reflects the higher throughput with VICO. We have also plotted the error bars (showing the maximum and minimum number of time slots consumed over the 20 runs with different client locations). Also notice that in the worst case, almost twice as many slots as with VICO, are needed with the simple scheduler that does not tune beamwidths or beam-angles.

Discussion: The reason for VICO's performance gains are the tuning of the beamwidth and beam-angle. Reducing the beamwidth provides the most gain since it improves the data rate while simultaneously improving spatial reuse. Simply tuning the beam-angle does provide an additional gain in spatial reuse; however, by itself it does not provide an increase in intensity (SNR) at the client and thus, an increase in data rate. By combining the two, VICO is able to offer high performance gains.

Comparing with the Optimal: Next, we compare the performance of VICO relative to the optimal. Due to the complexity involved in finding the optimal (with an exhaustive search) we consider a small scenario where we cast 4 LEDs and 8 clients in a room of size $3 \mathrm{~m} \times 3 \mathrm{~m} \times 3 \mathrm{~m}$. We perform an exhaustive search with all possible beamwidths and beamangles and find the best configuration that yields the minimum number of time slots. We then run VICO with the same client locations and find the number of slots consumed by the scheduler. We present the results with six such scenarios with randomly chosen client locations in Fig. 14. The results demonstrate that VICO is able to schedule the LEDs with the minimum number of slots in five of the six scenarios considered. In Scenario 3, it needs one additional slot compared to the optimal scheduler. We do recognize that this is a fairly small setting and inconclusive with regards to larger scenarios; however the exponential complexity of finding the optimal makes a study in larger settings prohibitive.

We wish to point out that even if the scheduling with VICO is sub-optimal, the reinforcement process wherein idle LEDs are used to increase the SNR at active clients restores (discussed next) some of the throughput lost due to suboptimal scheduling.

The contribution from idle LEDs: In the last functional module of VICO (Fig. 10), idle LEDs which are not transmitting in a time slot to avoid conflict to others try to reinforce the transmissions of adjacent LEDs to increase the SNR at 


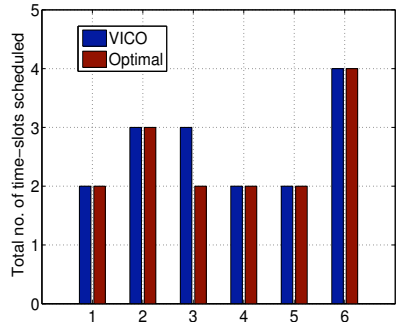

Fig. 14. Comparing the VICO scheduler with an optimal scheduler in six example scenarios.

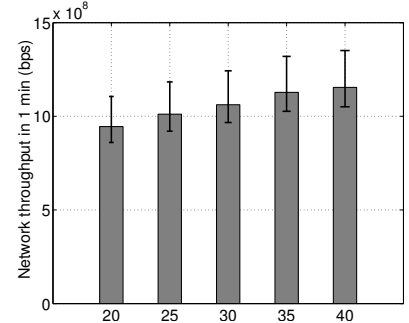

Fig. 15. Throughput variations with different numbers of clients.

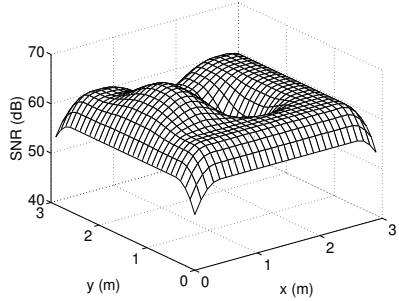

Fig. 16. Illuminance distribution with a specific scenario created by VICO in our OPNET simulations. a client. If using such reinforcement, an increase in SNR is possible, the data rate can potentially be increased at the corresponding clients. We perform 20 simulation runs with and without this reinforcement. (Note that the results reported earlier included the reinforcement component). We observe that the use of reinforcement with idle LEDs increases the network throughput by $31.58 \%$. Specifically, the throughput is $7.18 \times 10^{8} \mathrm{bps}$ for the case without VICO's final module, and is $9.45 \times 10^{8}$ bps with the module turned on.

VICO scales as the number of clients in the network increase: Next, we assess the performance of VICO with increased client (or node) density. We consider 5 scenarios wherein, the total number of nodes is $20,25,30,35$ and 40 , respectively. The room size is identical to what was considered earlier i.e., $4 \mathrm{~m} \times 4 \mathrm{~m} \times 3 \mathrm{~m}$. From the plot of the average throughputs shown in Fig. 15, we see that the network throughput increases with node density when we increase the number of nodes. This is because VICO is able to increase the number of simultaneously active clients in these scenarios. In other words, the number of links in each group is increased. As one might expect, if the densities are increased much further, the throughput does not increase anymore and a saturation point is reached. In fact, this point of diminishing returns is seen when we change the number of nodes from 35 to 40 .

The illumination variations with VICO are tolerable to the human eyes: Although we have earlier demonstrated that the variations in beamwidth and beam-angle considered results in illumination variations that are tolerable to the human eyes, we now verify this with VICO, in the settings considered in the simulations. While we have verified that all the scenarios lead to tolerable illuminations, we present a sample result from our OPNET simulations for a single scenario here, due to space constraints. In this scenario, we find that the LED 0 directs itself towards the LED 1's cell. LED 5 uses the larger beamwidth while the others use the smaller one (LED labels are shown in Fig. 6 for illustration). The results are in Fig. 16. We see from the figure that the minimum SNR is $49.2576 \mathrm{~dB}$ and while the average SNR is $61.0447 \mathrm{~dB}$ in the default setting. Looking back at Table I in Section III, we find that these are within acceptable limits of human tolerance.

\section{Conclusions}

We design VICO, a framework for configuring indoor VLC networks. This is one of the very first papers to consider higher layer protocol design and configuration issues in VLC settings. We first perform experiments on our PHY testbed and simulations in Matlab which demonstrates that tuning the LED beamwidths and beam-angles can effectively result in increased SNR and thus, data rates. We design a framework VICO that efficiently schedules LED to client transmissions while exploiting the ability to tune beamwidths and beamangles. Via extensive simulations we demonstrate the efficacy of VICO compared to schemes that do not exploit either or both of these degrees of freedom. VICO offers a 5-fold increase in throughput compared to a scheduler that does not exploit beamwidth adjustments or beam-angle tuning.

\section{REFERENCES}

[1] S. S. Haykin, "Communication Systems," 4th Edition, 2001.

[2] M. D. Audeh, J. M. Kahn and J. R. Barry, "Performance of PulsePosition Modulation on Measured Non-Directed Indoor Infrared Channels," IEEE Transactions on Communications, 1996.

[3] A. Shrestharinis, "Visible-light communication demonstrator: system modeling and analogue distribution network," Master thesis, 2009.

[4] K. Cui, G. Chen, Z. Xu and R. D. Roberts, "Line-of-sight visible light communication system design and demonstration," IEEE, IET international symposium on communications systems, networks, and digital signal processing: 2 nd colloquium on optical wireless communications, 2010.

[5] T. Komine and M. Nakagawa, "Fundamental analysis for visible-light communication system using LED lights," IEEE Trans on cosumer electronics, vol.50, no.1, February 2004

[6] J.M. Kahn and J.R. Barry, "Wireless infrared communications," Proc. of the IEEE, 1997.

[7] Francisco J. Lopez-Hernandez, R. Perez-Jimenez and A. Santamaria, "Ray-tracing algorithms for fast calculation of the channel impulse response on diffuse IR wireless indoor channels," Optical engineering, vol.39, no.10, 2000

[8] J. Grubor, O.C. Gaete Jamett, J.W. Walewski, S. Randel and K.D. Langer, "High-speed wireless indoor communication via visible light," ITG Fachbericht, pp.203-208, 2007.

[9] IEEE 802.15 WPAN Task Group 7 (TG7), "Visible Light Communication, http://www.ieee802.org/15/pub/TG7.html

[10] Z. Wu and T.D.C. Little, "Network solutions for the LOS problem of new indoor free space opticle system," IEEE, IET international symposium on communications systems, networks, and digital signal processing, 2010.

[11] S. Jivkova, B.A. Hristov and M. Kavehrad, "Power-efficient multispotdiffuse multiple-input-multiple-output approach to broad-band optical wireless communications," IEEE trans on vehicular technology, vol.53, no.3, May, 2004.

[12] C. Tangtrongbenchasil, "Ubiquitous optical wireless communication using optical micro-cell system," Ph.D. thesis, 2008.

[13] K. Jain, J. Padhye, V. Padmanabhan and L. Qiu, "Impact of inteference on multi-hop wireless network performance," ACM Mobicom, 2003

[14] E. Gelal, K. Pelechrinis, T.S. Kim, I. Broustis, S.V. Krishnamurthy and B. Rao, "Topology control for effective interference cancellation in multi-user MIMO networks," INFOCOM 2010, 2010.

[15] http://www.optekinc.com/datasheets/OPA742.PDF

[16] http://www.thorlabs.com/thorcat/13000/13054-S01.pdf

[17] http://www.thorlabs.us/thorProduct.cfm.partNumber $=F B 450-40$

[18] H. Yang, J.W.M Bergmans, T.C.W. Schenk, J. Linnartz and R. Rietman, "Uniform illumination rendering using an array of LEDs: a signal processing perspective," IEEE Trans on signal processing, vol.57, No.3, March 2009.

[19] "Indoor lighting standard" Nonresidential compliance manual, 2005.

[20] T. Jensen and B. Toft., Graph Coloring Problems, Wiley Interscience, 1995.

[21] N. Swangmuang and P. Krishnamurthy, "Location fingerprint analyses towards efficient indoor positioning," IEEE PERCOM, 2008.

[22] K. Chintalapudi, A.P. Iyer and V.N. Padmanabhan, "Indoor localization without the Pain," ACM MobiCom, 2010.

[23] OPNET User's Manual, http://www.opnet.com 